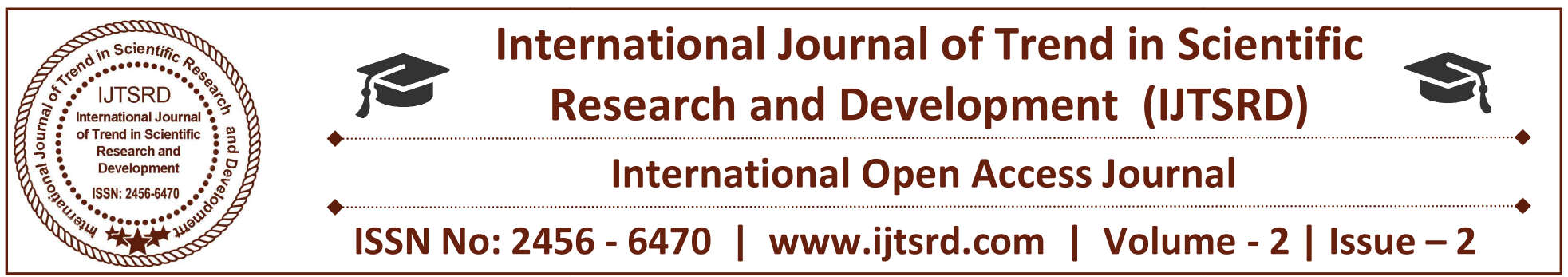

\title{
Analysis of Performance Quality of Next Generation Protocol: IPv6
}

\author{
Surbhi Khare \\ Assistant Professor, Priyadarshini Institute \\ of Engineering \& Technology, Nagpur
}

\author{
Ritesh Shrivastav \\ Assistant Professor, Anjuman College of \\ Engineering and Technology, Nagpur
}

\section{ABSTRACT}

Because of many-sided quality and size of ebb and flow Internet, how to secure the current speculation and diminish the negative impact to clients and specialist organizations amid the change from IPv4 to IPv6 is an imperative research theme for the future internet.IPv6 gives an extended deliver space to fulfill the future web prerequisites. A few methodologies for supporting Quality in best exertion IP systems have been produced and depicted in inquire about papers and articles and various research papers and ventures could show their feasibility with regards to IPv4 systems. Similar outcomes on a fundamental level ought to apply the instance of IPv6.In a web we realize that parcel postponement and misfortunes are the two essential parameters of IPv6 Quality measurements. In spite of the fact that these two parameters are not examined totally and furthermore as indicated by the best of our insight the development and advancement of extensive scale IPv6 deferral and misfortune execution has not beforehand investigated altogether. The initial step for the Quality tests was to settle on the tests would be performed what movement measurements would be utilized to assess them. The second step is to settle on the product apparatuses and applications that would be utilized to gauge, screen, and create the movement. In this paper we really think about IPv6 and IPv4 Quality metric issues like jitter, delay, misfortune execution, and way and passage disclosure. This paper exhibit and talk about the estimation philosophies and demonstrate that IPv6 ways have a more postponement and misfortune execution than their IPv4 partners.

Keywords: IPv6, Internet measurement, delay, Jitter, Packet loss, path and tunnel discovery

\section{INTRODUCTION}

IPv6 is the cutting edge IP convention to supplant the current IPv4.IPv6 gives an expanded address space and backings new web applications that require propelled highlights to give administrations like ongoing video, and sound. However IPv6 is as yet its in early stages and is seldom utilized .To qualify the IPv6 foundation, it is fascinating to look at the IPv6 and IPv4 estimations under the present system circumstances. There are numerous execution measurements propose to quantify the administration gave in a Quality empowered system. The objective of the IPv6 Quality movement is to demonstrate that methodologies for action is to show the methodologies which supporting for Quality in IPv4, easily relocated to the IPv6 condition. To compute the execution of IPv6 it scarcely requires estimations gathered after some time, joined with data about the jitter, delay, misfortune execution, way and passage disclosure. We have for the most part centered around IPv6 progress technologies[2] or distinguishing IPv6 organize issues in a double stack world by receiving IPv6 estimation devices within a couple of days $[3,4]$.As analyzed IPv4, IPv6 is in still sought after and it is just utilized at whatever point we like seldom. Because of this reality there is an absence of 
learning about the execution of end to end IPv6 correspondence. Amid the IPv6 Quality tests the accompanying parameters are utilized to redress the administrations gave by Quality like one path or round-trip delay, entomb - parcel delay variation(jitter), bundle misfortune, bundle reordering. We realize that as a rule vast scale sending of utilizations is adequately high. Hence we will contend that concentrate the development of huge scale IPv6 postponement and misfortune is imperative so as to take in all the execution of IPv6 organizes, and to give excellent administrations to future web applications. Jitter and deferral are the two essential Quality measurements and they will assume a vital part in the estimation of the execution of a web. Particularly for each source to goal combine we gather directing and one way postpone data utilizing IPv4 and IPv6 forms of the follow course and defer estimations and look at the steering defer information on a way - by-way premise.

In this paper we quickly examined the jitter and postpone execution in a web by utilizing such a large number of tests. The informational collection utilized as a part of this venture work contains dynamic estimations between an arrangement of around 26 test boxes supporting IPv6.

This paper shows an extensive clarification about the status of ebb and flow explore on IPv6 Quality issues, IPv6 Quality measurements, and demonstrates the possibility without bounds examine on IPv6 Quality measurements.

\section{RELATED WORK}

To the best of our Knowledge ,Hardly any work has quantified the IPv6 performance over time.Zhou.X.,Van Mieghem,Martin Jacobson ,Henk Uijterwaal[8]describe the IPv6 loss and delay performance evolution between the IPv6 and IPv4 networks. They presented and discussed the measurement methodologies and shown that IPv6 paths have higher delay and loss than their IPv4 counter parts.

Srivastava et al[11] describe the implementation of a test bed and the inter-connection between three domains using IPv6 -in- IPv4 static tunnels. They investigated performance issues (like) throughput, packet loss and delay) of aviation applications such as Controller to Pilot Data Link Communication using Diffserv on that IPv6-based backbone network. Their result suggested that Diffserv implementation and support in IPv6 has matured enough to provide stable and reliable Quality of Service (Quality) for the aviation applications.

Adam et al.[12] analyzed the issues of the implementation of the IPv6 service, IPv6 performance, the advantages of current transition technologies and the problems encountered. They also provided a performance comparison between three different transition mechanisms: IPv6 in IPv4 tunneling, 6PE tunneling (IPv6 over an IPv4 MPLS network) and dual stack in a local very high-speed broadband network.

Their experiments indicated that the current dualstack approach already achieves good performance.

Cho et al[13] measured both IPv6 and IPv4 round trip delays from two locations. Their results show that the majority of IPv6 paths have delay characteristics comparable to those of IPv4.

In [10], we compared and analyzed the hop count, end -to end delay, the delay variation like jitter between IPv6 and IPv4 in a month.

The IPv6 Quality [9] has also developed a comprehensive approach towards IPv6 Quality measurement objectives like

I. Development of a measurement device for IPv6. The measurement device inserts precise time stamp information when it captures the IPv6 packet. Each device has time synchronization functionality by GPS.

II. Development of a measurement server to collect captured IPv6 packet. It provides usage data Quality metrics, like delay, loss, jitter etc for IPv6 traffic by analyzing the collected information through the measurement device.

III. Generation of a set of guidelines for the possible application and further research of the IPv6 Quality measurement in different scenarios.

\section{RESEARCH METHODOLOGY}

In this paper first we reviewed the IPv6 Quality metrics, second we have gathered IPv6 data, which contains delay and loss measurements among a list of sites since 2006,trace.We have rune a tunnel discovery mechanism to identify the comparison between IPv6 paths and tunneled paths and study their differences. Lastly based on our observations we have 
listed some challenges for the measurement and analysis in IPv6 networks.

\subsection{The Comparison and Contrast between the IPv6 and IPv4 Quality metrics like delay and Jitter}

3.1.1. Types of Delay and Losses in IPv6 Quality Traffic

1. One way Packet Delay and One Way Packet Loss:
2. Reverse One Way Packet delay and one way Packet Loss:

In this paper our main intension is to compare and contrast the differences between IPv6 one way packet delay and one way packet Loss. The below Table-1 which shows the comparison between IPv6 One way packet delay and one way packet loss.

Type -1: One way packet delay and Packet Loss

Criterion-1: The below Table-1 shows the comparison between IPv6 Quality one way packet delay and one way packet loss.

Table-I: The Comparison and Contrast between IPv6 Quality metrics like one packet delay and one packet loss based on the Asymmetric path routing performance

\section{One Way Packet delay in IPv6 Quality}

1. The one way packet delay is the difference between the arrival time at destination and the departure time at the source. When a packet fails to arrive with in a reasonable period of time such as 10 seconds of time then the one way delay is undefined.

If the packet arrives within a reasonable period of time, the application at the destination takes the arriving time stamp from the kernel. By

Subtracting the two time stamps an estimate of the oneway delay can be computed.

2. The one way packet delay will become finite when the one way packet loss is exactly zero. Some times if the one way packet delay is undefined then also one way packet loss is exactly zero. As one way delay measurements require strict synchronization among the monitoring systems in order be able to reliably measure delay, clocks at the testing stations were synchronized using stratum 1 NTP server at ntps -1.0.cs.tu-berlin.de.

3.The Packet delay between two nodes is mainly due to various factors like

1. Number of Hops, 2.The load and capacity of the links, 3.The Policy routing decisions made along the path and even the way packets transported in the layer -2 architecture.

4.Some times minimum delay mainly reflects the propagation delay and transmission delay.

\section{One way Packet loss in IPv6 Quality}

1. Understanding the one way packet loss from a Source to a Destination is motivated by the fact that excessive packet loss i.e. relative to some threshold value could degrade the perceived quality of certain real -time applications.

One way delay is defined as the time needed by a packet to be transmitted and fully received by the Destination. The overall time consists of the propagation delay.

2. One way packet loss is exactly zero then one way delay is finite and the one way delay is undefined. Packet loss occurs where network traffic fails to reach the destination with in a reasonable period of time. Losses may depend on the various factors like congestion of the network, changes in paths between the source and destination or incorrect routing.

No Delay 
Criterion-2: The below Table-2 shows the comparison between IPv6 Quality delay (Packet Loss) V/s Throughput w.r.t. transport layer Protocols

\section{Table-II: The Comparison between Packet delay v/s Throughput}

\begin{tabular}{|l|l|}
\hline \multicolumn{2}{|l|}{ Packet Delay(Loss) } \\
$\begin{array}{l}\text { 1. Whenever packet delay is high then it is very } \\
\text { difficult to sustain high throughput. }\end{array}$ & $\begin{array}{l}\text { 1. Whenever the packet delay is low then only } \\
\text { transport layer protocols like UDP or TCP sustain } \\
\text { High throughput. }\end{array}$ \\
\hline
\end{tabular}

Criterion-3: The below Table-3 shows the Differences between packet loss and Delay w.r.t. Transmission/Receiving

\section{Table-III: The Comparison between Packet loss v/s Delay.}

Here packet loss is calculated as the portion of the packets transmitted but not received by the destination compared to the total number of packets transmitted. Large values of packets loss usually shows highly congested networks or frequent sharp increases in the traffic load.

\section{DISCUSSIONS}

As demonstrated earlier, the IPv4 delay performance shows a slight improvement over the 1 year. No such trend is seen for either IPv6 native or IPv6 tunnel paths. Instead, we see big variations in the delay performance. Concerning loss, IPv4 again performs best. However, when comparing IPv6 native and tunnel paths, no certain difference can be demonstrated. Packet Loss can be summarized as follows.

It is always difficult to explain all these behaviors without a detailed view on all involved networks. However, some causes of the longer delay for IPv6 can be explained as follows:

(1) The lack of routers with IPv6 hardware-optimized implementation. Hardware-based IPv6 tunneling implementations are virtually non-existing today. However, some software-based IPv6 routers can perform quite Well [16].

1. Less optimal paths are used for IPv6, especially when tunnels are used.

2. There are different or fewer peering agreements for IPv6 between ISPs.

3. Network management and monitoring of IPv6 networks are not as advanced as for IPv4 networks. ISPs do not invest equally on IPv6 network management as they do on IPv4 Also, the experience with IPv4 is larger (e.g. more traffic engineering).

4. IPv6 has a lower priority in the routers. IPv6 is still seen as experimental and should never degrade the performance of the more important IPv4 traffic.

It is expected that IPv4 and IPv6 will coexist for a while, and that IPv6 tunnels play a key role in the transient phase. Software-based routers and tunnels provide a quick way to deploy IPv6. The drawback, on the other hand, is that IPv6 tunneling degrades the traffic performance mainly in terms of larger delay. Clearly, our results suggest that for a better IPv6 quality, we should only use native IPv6 and hardwarebased routers everywhere. Nevertheless, the performance quality of software-based routers and tunnels is still acceptable for a successful transition phase 


\section{CONCLUSION}

A detailed measurement study of the delay and loss evolution in IPv6 networks based on the implemented work has been presented. Although IPv6 will replace IPv4 in the future, it expected that IPv4 and IPv6 hosts will coexist for a substantial time during the steady migration from IPv6 to IPv4.In this paper describes the depth study on the comparison and contrast between IPv4 and IPv6 Quality metrics like Packet Loss Performance, Packet delay, jitter, tunneling discovery etc. In this paper the development of a measurement server to collect captured IPv6 packet. It provides usage data and Quality metrics (delay, loss, jitter and so on) for the IPv6 traffic by analyzing the collected information through the measurement device. It also coordinates for the generation of a set of guidelines for the possible application and further research of the IPv6 Quality measurement in different scenarios. Concerning the delays over one day, native IPv6 paths have small 2.5 percentile and median end-to-end delay, and comparable delay to their IPv4 counterparts. IPv6 tunnel paths have relatively large 2.5 percentile and median end-to-end delay, and about half of the paths have significantly more delay compared with their IPv4 counterparts. The worst performance came from IPv6-in-IPv4 tunnels. For the 97.5 percentile delay, IPv4 by far outperforms IPv6 for both native and tunnel IPv6 paths.

\section{REFERENCES}

1. Dr.Manjaih.D.H. Hanumanthappa.J,2008,A Study on Comparison and Contrast between IPv4 and IPv6 Feature sets. In Proceedings of ICCNS'08, 2008, Pune,297-302.

2. Dr.Manjaih.D.H.,Hanumanthappa.J.,2008,Transiti on of IPv4 Network Applications to IPv6 Applications, In Proceedings of ICETiC09,2009,S.P.G.C. Nagar,VirudhaNagar-626 001, TamilNadu ,INDIA- 35-40.

3. Dr.Manjaih.D.H.Hanumanthappa.J.,2009,IPv6 over Bluetooth :Security Aspects, Issues and its Challenges, In Proceedings of NCWNT09,2009,Nitte-574 110,Karnataka ,INDIA - 18-22
4. Dr.Manjaih.D.H.,Hanumanthappa.J.,2009,Econo mical and Technical costs for the Transition of IPv4 -to-IPv6 Mechanisms [ETCTIPv4 to ETCTIPv6], In Proceedings of NCWNT09,2009,Nitee-574 110,Karnataka,INDA-12-17.

5. Dr.Manjaih.D.H. Hanumanthappa.J.,2009,Smooth porting process scenario during the IPv6 transition, In Proceedings of IACC'09, March 6-7 ,Patiala,Punjab,INDIA.

6. S.Deering and R.Hinden "Internet Protocol Version 6(IPv6) Specification", RFC 2460, December 1998.

7. Durand A. managing $100+$ million IP addresses .The North America Network operator's group(Nanog 37), meeting, San Jose , CA,U.S.A. „June 2006 .

8. IPv6 network administration guide.

9. Zhou.X, Van Mieghem,, Martin Jacobson, Henk Uijterwaal, IPv6 delay and loss performance evolution, International Journal of Communication Systems, published online 28, December 2007.

10. IPv6 QualityM, Issues and Solutions, Allessandro Bassi, Itachi Europe SAS.

11. David Brumley and Dan Boneh. "Remote timing attacks are practical", In Proceedings of the $12^{\text {th }}$ USENIX Security Symposium, pages 1-14. Usenix, 2003.

12. Martim Carbone, Weidong Cui, Wenke Lee, Marcus Peinado, and Xuxian Jiang. "Mapping kernel objects to enable systematic integrity checking" ACM Conference on Computer and Communications Security, 2009. 